\title{
Full-body illusions and minimal phenomenal selfhood
}

\author{
Olaf Blanke ${ }^{1,2}$ and Thomas Metzinger ${ }^{3,4}$ \\ ${ }^{1}$ Laboratory of Cognitive Neuroscience, Ecole Polytechnique Fédérale de Lausanne (EPFL), Swiss Federal Institute of Technology, \\ 1015 Lausanne, Switzerland \\ ${ }^{2}$ Department of Neurology, University Hospital, 1214 Geneva, Switzerland \\ ${ }^{3}$ Philosophisches Seminar, Johannes Gutenberg-Universität Mainz, 55099 Mainz, Germany \\ ${ }^{4}$ Frankfurt Institute for Advanced Studies, Johann Wolfgang Goethe University, 60438 Frankfurt am Main, Germany
}

\begin{abstract}
We highlight the latest research on body perception and self-consciousness, but argue that despite these achievements, central aspects have remained unexplored, namely, global aspects of bodily self-consciousness. Researchers investigated central representations of body parts and actions involving these, but neglected the global and unitary character of self-consciousness, the ' $I$ ' of experience and behaviour. We ask, what are the minimally sufficient conditions for the appearance of a phenomenal self, that is, the fundamental conscious experience of being someone? What are necessary conditions for self-consciousness in any type of system? We offer conceptual clarifications, discuss recent empirical evidence from neurology and cognitive science and argue that these findings offer a new entry point for the systematic study of global and more fundamental aspects of self-consciousness.
\end{abstract}

\section{Introduction}

Recent years have seen a flood of research into self-consciousness, and a renewed interest in its bodily foundations [1,2]. In philosophy of mind, there is widespread agreement that the core of the problem consists of understanding the pre-reflective bodily foundations of phenomenal selfhood (defined here as all those levels which are independent of explicit cognition and linguistic abilities, and which give rise to the subjective experience of being a self), but which can function as enabling conditions for a conceptually mediated, cognitive first-person perspective and high-level social cognition [3-8]. Prominent candidates are associated with notions such as 'agency' [9-11] and 'embodiment' (see Glossary). Here, we argue that from a strategic point of view, future research should focus on what we call 'minimal phenomenal selfhood' (MPS), which is related to the concept of embodiment and the simplest form of self-consciousness.

To illustrate this, we review how researchers have investigated embodiment and the bodily foundations of self-consciousness through the sense of ownership and identification with the body as a whole (phenomenally experienced 'mineness'), self-location (centeredness of the conscious model of reality) and the first-person perspective. Next, we introduce a conceptual distinction

Corresponding authors: Blanke, O. (olaf.blanke@epfl.ch); Metzinger, T. (metzinger@uni-mainz.de). between the conscious experience of 'ownership for body parts' (as studied in the rubber-hand illusion (RHI) [12]) and the experience of 'global ownership' (as studied in the full-body illusion [13]). We argue that only the latter enables investigations of MPS, with MPS being a clearer concept to guide future research. From a theory-of-science point of view, and given a complex domain such as human self-consciousness, we propose that an optimal strategy is likely to be the (initial) isolation of the simplest form of the target phenomenon. MPS is a phenomenal property, namely the conscious experience of being a self. It is the experience of being a distinct, holistic entity capable of global self-control and attention, possessing a body and a location in space and time.

\section{Glossary}

Agency: global motor control, including the subjective experience of action, control, intention, motor selection and the conscious experience of will. It involves the representation of goal-states.

Cognitive 1PP: arguably, this appears when a system possesses a concept of the strong 1PP and is able to apply this concept to itself (i.e. it has an abstract and active mental representation of itself as a subject of experience, which includes a special form of cognitive self-reference) [3,6-8]. The cognitive 1PP presupposes the capacity of self-reference in a more narrow sense, that is, to mentally refer to oneself using a concept such as ' $I$ ', for example by consciously thinking I-thoughts such as 'I am happy' or 'I myself am a thinking thing' or 'I myself am a subject of experience'. Many organisms might have phenomenal self-models [6,23] (MPS), but perhaps only humans have selfconcepts.

Embodiment: a position in cognitive science and the philosophy of mind that emphasizes the relevance of sensorimotor skills for general intelligence, the situtatedness of cognition and the role that the body has in shaping the mind, plus the subjective experience of using and 'having' a body. The concept is used in many different ways. Different functional levels exist (Thomas Metzinger [2007], Self models. Scholarpedia: www.scholarpedia.org/article/ Self_models).

Self-identification: the degree to which an organism identifies with the content of a global body representation $[7,24]$.

Self-location: a determinate volume in space, normally localized within the bodily boundaries as represented. The origin of the weak 1PP is localized within this volume ('embedding principle'). Self-location is also necessarily spatiotemporal self-location (because it includes the 'now', a determinate position in time as represented). This is not discussed here [6].

Strong 1PP: this occurs when the model of the organism as a whole, given through MPS, is represented as being directed at an object component (including, potentially, the body itself). We speculate that a strong 1PP necessarily (and minimally) involves the additional experience of being able to control the focus of attention.

Weak 1PP: a purely geometrical feature of an egocentric model of reality, most often referred to as a visuospatial model. A weak 1PP includes a spatial frame of reference, plus a global body representation, with a perspective originating within this body representation. A visual (or auditory) weak 1PP possesses a centre of projection, which functions as the geometrical origin of the 'seeing' (or 'hearing' etc.) organism's embodied perspective. 


\section{MPS: conceptual foundations}

MPS can be analyzed on phenomenological, representational and functional levels of description. Central defining features are, (i) a globalized form of identification with the body as a whole (as opposed to ownership for body parts), (ii) spatiotemporal self-location and (iii) a first-person perspective (1PP). It is important to differentiate between a weak and at least two stronger readings of 'first-person perspective', with MPS being partly constituted by the weak 1PP, and a necessary (but not sufficient) condition for stronger versions [14-19]. A weak $1 \mathrm{PP}$ is a purely geometrical feature of a perceptual or imagined model of reality possessing a point of projection functioning as its origin in sensory and mental processing, but is not linked with theoretically more charged notions such as 'subject of experience' (the conscious self) or 'epistemic subject' (knowing self) [14-19]. A strong 1PP appears when the system as a whole is internally represented as directed at an object component, for example a perceptual object, an action goal as internally simulated or perhaps the body as a whole. A strong $1 \mathrm{PP}$ is exactly what makes consciousness subjective: the fact that a system represents itself not only as a self but also as 'a self in the act of knowing' [20] (Box 1). This means that the organism represents itself as representing in real time. It co-represents the representational relation during the ongoing process of representation: a phenomenal self as subject appears exactly when the organism possesses a phenomenal model of the intentionality relation [6,7,9,21,22] (Thomas Metzinger [2007] Self models. Scholarpedia: www.scholarpedia.org/article/Self_models). There is an even stronger 1PP, which implies the possession of the concept of a subject of experience, and the additional feature of a given system to mentally apply this concept to itself ('cognitive 1PP'), which will not be discussed here [3,6-8].

Having MPS is a necessary condition for the strong (and the cognitive) $1 \mathrm{PP}$, but not for the weak $1 \mathrm{PP}$ because the weak 1PP is itself a constituting factor for MPS. A conscious body model defines a volume within a spatial frame of reference (the dimension of 'self-location') within which the origin of the weak 1PP is localized (for temporal aspects of self-location see Refs [6,18]). Because this conscious body model is phenomenally transparent because it cannot introspectively be recognized as a model, we fully identify with its representational content and are consciously present as bodily selves (self-identification) [6,7,23,24]. It is this bodily self or MPS which is altered and abnormal during out-of-body experiences [23-25], heautoscopy (see Box 2) $[15,26,27]$ and experimentally manipulated during the full-body illusion [13,28]. If parts of the conscious bodymodel become phenomenally opaque, that is, if they are experienced as a representation (Box 2), the dimension of self-identification and consequently MPS can be weakened.

The essence of selfhood, the representational content of MPS, is that it indicates to the system that a specific state in its internal neural dynamics has been reached, namely an integrated functional state, which for the first time makes the body available for attention and global control. MPS, characterized by self-identification, self-location, and weak 1PP, is the outcome of this process on the level of conscious experience. MPS, the simplest form of selfhood, is a representation of the entire body, being functionally available for global control: a form of abstract (yet nonpropositional) knowledge about oneself and provides information about new causal properties. MPS or any selfrepresentation are not static internal copies of some mysterious thing or individual substance ('the' self), but only ongoing processes of tracking and controlling global bodily properties - for example with the help of an explicit, integrated body-model. 'Global control' is a process in which a system controls global properties of itself (as opposed to local properties). A global property is one that can only meaningfully be ascribed to a given system as a whole, often because it requires a new level of analysis defined by a different logical subject. A drop of water is wet, but none of the individual $\mathrm{H}_{2} \mathrm{O}$ molecules has the property of 'wetness'. Global properties of the human body are the entire body's surface or translation (linear passive motion)

\section{Box 1. Conceptual distinctions}

\section{Necessity}

A condition ' $A$ ' is said to be necessary for a condition ' $B$ ', if (and only if) the non-existence or non-occurrence of ' $A$ ' guarantees (or brings about) non-existence of non-occurrence of ' $\mathrm{B}$ '. We propose that MPS is a necessary condition for the strong and cognitive 1PP.

\section{Sufficiency}

A condition ' $A$ ' is said to be sufficient for a condition ' $B$ ', if (and only if) the existence or occurrence of ' $A$ ' guarantees (or brings about) existence or occurrence of ' $B$ '. The existence of a conscious, transparent and global body-representation available for attentional access is a sufficient condition for MPS.

\section{Necessary conditions that are not sufficient}

There could be sets of conditions, which are individually necessary, but not jointly sufficient. We claim that spatiotemporal self-location, self-identification and weak 1PP are necessary and sufficient for MPS. If there are further necessary conditions, then our claim is false.

Sufficient conditions that are not necessary

There could be various sets of (jointly) sufficient conditions, none of which is individually necessary. Weak 1PP, self location and self identification are jointly sufficient for MPS. Perhaps one or more of them are not individually necessary.

\section{Causally enabling conditions}

Conditions that have a causal role in bringing about a phenomenon, but are not strictly necessary (and frequently, if taken by themselves, not even sufficient). In striking a match, the presence of oxygen is a nomologically necessary condition for ignition, whereas my hand executing the motion of scratching it is only causally enabling because this motion could also be carried out by a machine. The temporally preceding experience of agency and of global motor control (e.g. in childhood) causally enables MPS in practically all humans, but it is not causally necessary (because there are other causal routes to the same phenomenon). Out-of-body experiences of neurological origin and the selective experimental manipulation of the dimensions of self-location and self-identification demonstrate that agency only belongs to the causal background for MPS. Therefore, it is not a metaphysically necessary condition for the target phenomenon to come into existence, nor part of the minimal supervenience base. Conceptually, what constitutes MPS is something different or less that could also take place in a disembodied, non-neural, artificial system possessing no effectors. 


\section{Box 2. Illusory global own body perceptions}

Illusory own body perceptions are characterized by seeing a second own body in extracorporeal space $[15,24,47-49]$. We describe weak $1 \mathrm{PP}$, self-location and self-identification for each illusory perception.

\section{Out-of-body experience}

Self-location and the origin of the weak $1 \mathrm{PP}$ are at the position of the illusory body at a position that is most often elevated with respect to the position of the physical body as represented $[15,48]$. Self-location and the origin of the weak 1PP are never at the position of the physical body as represented, and out-of-body experiences are associated with a sense of disembodiment (the experience that the subject of conscious experience is localized outside the person's bodily borders) [23]. Selfidentification refers to the illusory body, not the physical body as represented. All three dimensions of the MPS are pathological. The strong 1PP is also pathological because out-of-body experiences are characterized by an error through misidentification (consistent selfidentification with the incorrect content of a global body representation $[55,63])$. Out-of-body experiences are highly realistic, and most often there is no insight into the illusory character of the phenomenon [23].

\section{Autoscopic hallucination}

Self-location and the origin of the weak 1PP are at the position of the physical body as represented. There is no sense of disembodiment and self-identification refers to the physical body as represented. All three dimensions of the MPS are normal $[23,26]$. The person sees an illusory own body in front of him, but has preserved insight into the illusory character of the phenomenon.

\section{Heautoscopy}

Self-location and the origin of the weak 1PP are either at the position of the physical body as represented or at the position of the illusory body as represented or at both positions at the same time [50]. Both MPS dimensions can change their experienced position (between the position of the illusory and the physical body as represented) and this can occur in rapid alternation [23]. The origin of auditory and visual weak $1 \mathrm{PP}$ can be dissociated and experienced at different positions. The position of self-location and weak 1PP can also differ [23]. Heautoscopy is sometimes associated with 'bi-location' (the experience that self-location and the origin of the weak $1 \mathrm{PP}$ are simultaneously at different positions). Self-identification either refers to the illusory body as represented, the physical body as represented or to both, in alternation or simultaneously. Heautoscopy is often associated with the sensation of 'being split in two selves' [23]. All three dimensions of the MPS are pathological as is the strong 1PP. Heautoscopy is highly realistic and the person has most often no insight into the illusory character of the phenomenon. as opposed to partial properties such as the skin of the fingertip or hand translation (Box 1). The possession of MPS, of a strong or even cognitive 1PP are also global properties, they are not things that individual parts can possess. If the integration processes leading to the phenomenal self-model $[6,8]$ are disturbed (if certain states are isolated from global control), then disturbances of bodily self-consciousness will appear and these might affect certain body parts, in addition to the entire body. The same principle also applies to specific emotional or cognitive states: they become your feelings and thoughts by being embedded into the phenomenal self-model - which, in standard situations, is already more complex than mere MPS $[6,8,9]$. MPS and its dimensions have the potential to describe the phenomenology associated with such functional deficits in an adequate and conceptually fine-grained manner.

\section{Partial versus global ownership}

Ownership for a body part can break down after damage to right temporo-parietal cortex, this is known as somatoparaphrenia [29-31]. Somatoparaphrenic patients most often misattribute their contralesional hand as belonging to another person, mostly a familiar person (e.g. a doctor, nurse or friend) [31]. Other somatoparaphrenic patients suffer form the opposite pattern and self-attribute other people's hands when these are presented in their contralesional hemispace [29]. Somatoparaphrenia affects only the contralesional (mostly left) hand (or foot), and never ipsilesional body parts. Somatoparaphrenia should be distinguished from other bodily symptoms reflecting disturbed partial ownership and/or agency [31].

Errors in partial ownership have also been induced experimentally in healthy subjects during the RHI by using a fake hand and multisensory conflict [12]. Viewing a fake hand being stroked by a paintbrush in synchrony with strokes applied to one's own corresponding (but occluded) hand can induce the tactile illusion that the fake hand is touched. Subjects might also report ownership for the fake hand. In addition, there is a change in perceived hand position (the hand is experienced as though it is displaced towards the fake hand's position). The RHI is abolished when the stroking is asynchronous [32,33]. Brain imaging studies associated the RHI with bilateral activations in several brain regions [34,35], yet this is only partly compatible with data on somatoparaphrenia that highlight the importance of temporo-parietal cortex and the right hemisphere.

Despite their importance for the study of body ownership, somatoparaphrenia and RHI are only associated with changes in partial ownership and do not enable the investigation of MPS as the most fundamental sense of selfhood. MPS is associated with global, fully embodied self-consciousness and is experienced as a single feature, namely a coherent representation of the whole, spatially situated body - and not as multiple representations of separate body parts (i.e. finger [33,36], hand [12] or hemibody [37]). Accordingly, MPS is not altered in somatoparaphrenic patients and not manipulated in healthy subjects during the RHI because these studies do not experimentally manipulate self-location, self-identification or the weak 1PP [38].

Neural mechanisms of partial ownership and selfidentification are also likely to differ. Neurons in primary somatosensory cortex (area $3 b$ ) have tactile receptive fields that respond preferentially to stimuli applied to a specific body part (i.e. fingertip [39]). Neurons in higher-tier somatosensory cortex (i.e. area 5) have larger receptive fields responding to cutaneous stimuli applied to hands, but also proximal parts of an extremity or the back [40-42]. Neurons in ventral intraparietal area (area VIP) [43] might encode the entire or large parts of the body surface [44]. Latter cells will discharge to a cutaneous stimulus that is applied anywhere on the body surface. We argue that cells in area VIP and 5 are of importance for self-identification and other MPS-dimensions, whereas cells representing 
fingertips or hands are involved in partial ownership and related aspects. Another sensory system important for global body representation and MPS is the vestibular system because it encodes translation and rotation of the body in addition to orientation with respect to gravity $[45,46]$.

\section{Abnormal MPS in neurology}

MPS breaks down in neurological patients with illusory global own body perceptions, called autoscopic phenomena $[23,27,37,47-49]$, that are characterized by seeing a second own body in extracorporeal space: autoscopic hallucination, heautoscopy, and out-of-body experience (Figure 1) (Box 2). Autoscopic phenomena are associated with multisensory disintegration and can be caused by focal brain damage (Figure 2) [26,27]. Recent neuroanatomical analysis indicated that autoscopic hallucinations are preferentially caused by damage to right parieto-occipital or right temporo-occipital cortex, whereas heautoscopy and out-of-body experience are caused by damage to temporoparietal cortex of the left and right hemisphere, respectively $[27,47]$.

Autoscopic phenomena show that not only identification with and localization of body parts but also the conscious representation of the entire body and MPS can be disturbed [45] (Box 2). Patients with autoscopic hallucinations experience themselves to be localized at, and to see from, the physical body's position as represented. Selfidentification is referred to the global representation of

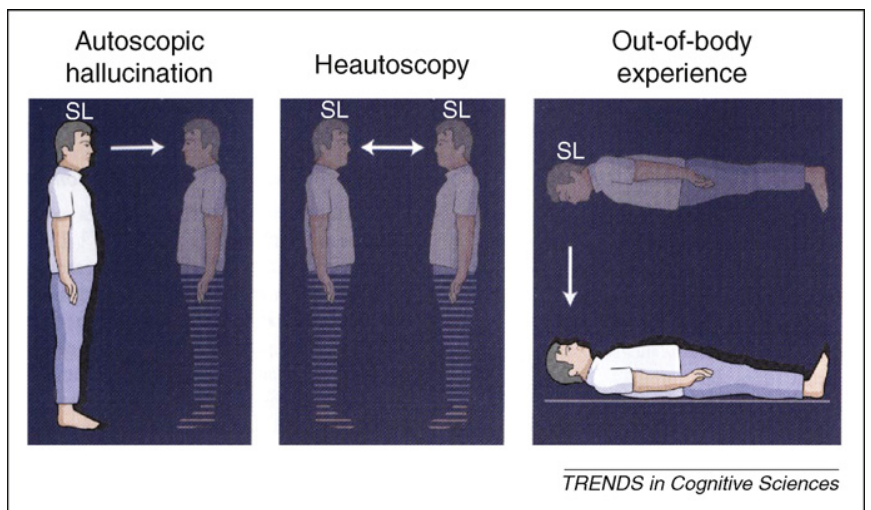

Figure 1. Phenomenology of three illusory own body perceptions (autoscopic phenomena) leading to alterations of MPS. Autoscopic phenomena are defined as illusory own body perceptions characterized by seeing a second own body in extracorporeal space. The three autoscopic phenomena differ with respect to selflocation and the weak 1PP. During autoscopic hallucinations, the person sees a second illusory own body from the habitual weak 1PP with normal self-location (at the physical body as represented). During an out-of-body experience, self-location and the weak $1 \mathrm{PP}$ are at the position of the illusory body as represented (at a position that is most often elevated with respect to the position of the physical body as represented). Heautoscopy is an intermediate form and self-location and the origin of the weak 1PP are either at the position of the physical body as represented or at the position of the illusory body as represented or at both positions. Self-identification either refers to the illusory body as represented, the physical body as represented or to both. For each autoscopic phenomenon, the physical position of the patient's body is schematically represented by the solid body and that of the illusory body by the transparent body. The location and direction of the visual weak $1 \mathrm{PP}$ is indicated by an arrow. Self-location is where the patient has the impression that they are located (indicated by ' $S L^{\prime}$ '). The patient has the impression to see the environment from the physical body as represented in the case of autoscopic hallucination, from the illusory body as represented in the case of out-of-body experience and alternatively or simultaneously from the physical and the illusory body as represented in the case of heautoscopy (after [23]). For further detail, see Box 2.

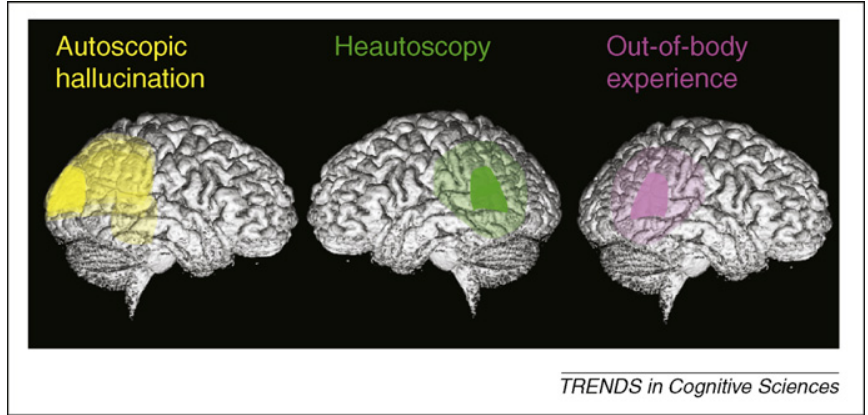

Figure 2. Location of brain damage for each of the three illusory global own-body perceptions (autoscopic phenomena) leading to alterations of MPS. Analysis of lesion overlap of brain damage in patients with autoscopic hallucinations shows a centre of damage in the right parieto-occipital or right temporo-occipital cortex (lesion reconstruction was estimated based on 7 patients [26]). For out-of-body experiences, damage was localized primarily in right temporo-parietal cortex ( $\mathrm{n}=$ $12[27,51])$ and for heautoscopy in left temporo-parietal cortex $\left(n=10^{28}\right)$. Lesion overlap was estimated based on magnetic resonance imaging data (acquired by of one of the authors) and detailed neuroradiological and neuropathological data reported in the literature. Although single cases of each type of autoscopic phenomenon have been reported after damage to the right or left hemisphere, we indicated in the figure the hemisphere of predominant involvement in autoscopic hallucination (right), heautoscopy (left) and out-of-body experience (right).

the physical body (Figure 1). All three MPS dimensions (self-identification, self-location, weak 1PP) are normal. Heautoscopy is characterized by abnormality in all three MPS dimensions. Self-location and weak 1PP are either at the position of the physical body as represented or at the position of the illusory body as represented, or at both. Moreover, self-location and weak 1PP might change their experienced position (between the position of the illusory and the physical body as represented) and this can occur in rapid alternation. Self-identification either refers to the illusory body as represented, the physical body as represented or to both in alternation [23,27,49] (Box 2). In out-of-body experiences the weak $1 \mathrm{PP}$, self-location and self-identification with an illusory body as represented at an extracorporeal position are complete: self-location and weak 1PP are localized outside one's bodily borders and people have the experience of seeing their body from this disembodied location (Figure 1). Out-of-body experiences are associated with a sense of disembodiment and people always self-identify with the illusory body as represented. These neurological autoscopic phenomena demonstrate how global bodily experience varies along MPS dimensions. In particular, there seems to be a continuum between transparency and opacity, which determines how realistically the content of a body representation is experienced and how strongly the dimension of self-identification is expressed [6,7].

To summarize, heautoscopy and out-of-body experiences are examples of abnormal MPS, also extending to impairments of the strong 1PP (characterized by selfidentification with an incorrect content of a global body representation). Although MPS is modified differently in heautoscopy and out-of-body experiences, MPS does persist in both conditions (a situated body as represented is always the object of self-identification). Autoscopic hallucinations are also conceptually relevant (but as neurological control conditions of MPS) because, despite their neurological origin (presence of an illusory own body perception, distinct neural correlate), they are characterized 
by normal MPS. These data also reveal how brain mechanisms for the dimensions of self-identification, selflocation and weak 1PP are linked because changes in weak $1 \mathrm{PP}$ and self-location are often experienced as spatially congruent: if the geometrical point of origin for the weak $1 \mathrm{PP}$ is experienced at an extracorporeal position as in heautoscopy, self-location is also experienced at this position. Self-identification is often strongest for this position as well.

\section{Further observations on MPS dimensions in autoscopic phenomena}

In the majority of such autoscopic phenomena, MPS is characterized by a single visual weak $1 \mathrm{PP}$, spatially overlapping self-location and non-ambiguous self-identification. Although under normal conditions humans experience a single visual weak $1 \mathrm{PP}$, autoscopic phenomena indicate that conscious experience can also be characterized by the absence of a unitary visual weak $1 \mathrm{PP}$, or by two simultaneous or rapidly alternating visual weak 1 PPs. This is the case in patients with heautoscopy who claim to jump back and forth between two spatially distinct points of view $[23,50]$. Other patients can describe an auditory weak 1PP that is spatially distinct from their simultaneously present visual weak 1PP [23]. Moreover, the weak 1PP (auditory or visual, or both) does not always overlap with the location of the bodily self. The origin of the weak 1PP can be spatially dissociated from self-location as indicated by a recent study using electrical brain stimulation [51]. Especially during heautoscopy (with rapidly alternating or two simultaneous centres of projection for the weak 1PP), patients can report to 'be split in two parts or selves', feeling as if 'I were two persons' [52] or a 'split personality' [53]. This indicates that a breakdown of a single weak 1PP or the loss of spatial congruency between the weak 1PP and self-location has important consequences for MPS and the strong 1PP [27]. With respect to self-identification, these observations indicate that there seems to be a transient, but nevertheless complete, heautoscopic dissociation into two objects of identification, leading to a brief double instantiation of MPS (two selves) and the strong 1PP (a reduplication of the experience of being directed) that does not occur in any other form of autoscopic phenomenon.

\section{Experimenting with MPS}

Recent studies have extended clinical data on illusory global own body perceptions and studied MPS experimentally, refining the seminal self-observations made at the end of the 19th century by G.M. Stratton (Figure 3a) [54]. By exposing participants to conflicting multisensory bodily cues by means of mirrors, video technology or simple virtual reality devices $[13,28,55,56]$, these authors experimentally manipulated MPS. In particular, the presentation of stimuli in virtual reality set-ups has enabled systematic manipulations of different sensory dimensions including somatosensory, visual and perspectival cues.

In a study by Lenggenhager and colleagues [13], an experimental protocol similar to that in the RHI [12] was developed for the full body (full-body illusion; Figure $3 b)$. A video camera with a $3 \mathrm{D}$-encoder was placed (a)

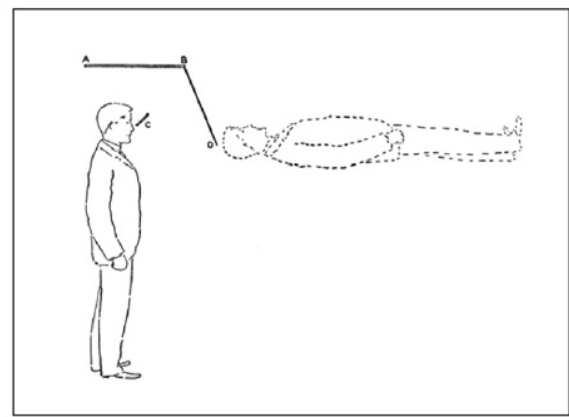

(b)

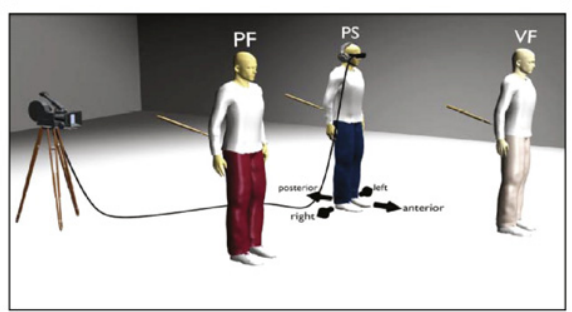

(c)

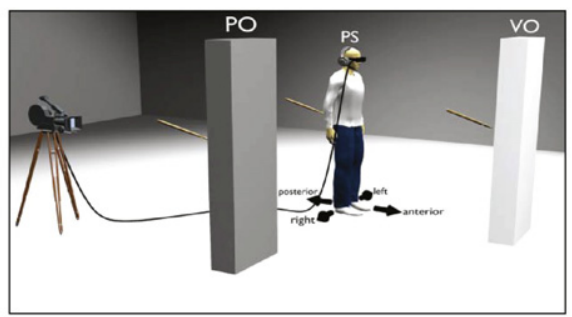

(d)

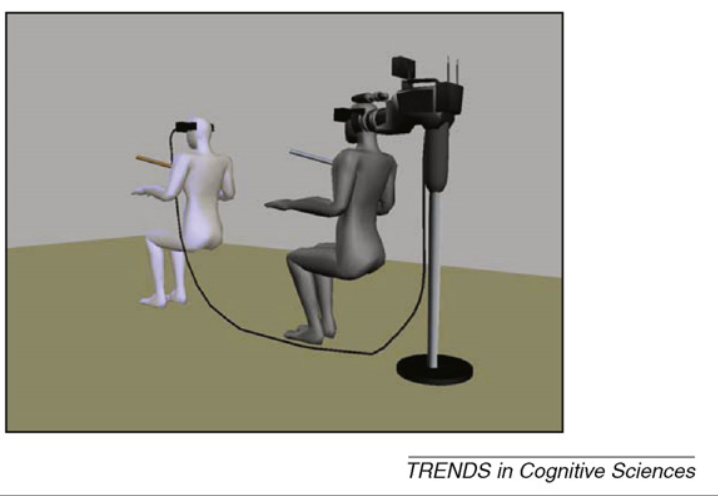

Figure 3. Experimenting with MPS using the full-body illusion. (a) Experimental setup of the seminal self-observations made at the end of the 19th century by G.M. Stratton [54]. Through the use of a portable mirror system, Stratton [54] was able to project an online image of his body in his anterior peripersonal space while he was wandering through the countryside around Berkeley. He described changes in selflocation and self-identification that progressively increased over the three days of exposure. (b,c) Experimental set-up of the full-body illusion [13]. Participant (physical subject $[\mathrm{PS}]$ in dark blue trousers) sees through a head-mounted display (HMD) either a virtual fake body (VF, pink trousers) or a virtual non-corporeal object (VO, light gray) being stroked synchronously or asynchronously with his back. Dark colours indicate the actual location of the physical body/object whereas light colours represent the virtual body/object seen on the HMD. Participants' self-identification and self-location was modified, leading to self-identification only with the virtual body and a drift in self-location towards the virtual body to an extracorporeal position, especially during synchronous stroking. (d) Alternative experimental set-up of the full-body illusion [28]. Participant sees through a HMD the own body being stroked synchronously or asynchronously, yet stroking is applied at the chest (unseen) and in front of the camera. This induced the experience of being at the position of the camera that was behind the subjects' body and diminished selfidentification with the virtual body during synchronous stroking.

behind the subject and relayed to a head mounted display. Tactile stimulation (stroking) was applied on the back and the visual information related to this stimulation was systematically manipulated by displaying it as either synchronously or asynchronously with the tactile sensation. 
Questionnaire scores were used to quantify self-identification and perceived body position (drift) to quantify selflocation. When the participants saw their body in front of themselves, being stroked synchronously (with their own back), they felt as if the virtual body was their own (illusory self-identification) and showed drift towards it (illusory self-location). Both full-body illusion measures were also observed for a fake body (Figure 3c), but not (or less so) for a rectangular object. The full-body illusion was abolished during asynchronous stroking. Ehrsson [28] used a similar set-up but stroked subjects on their chests (hidden from the view of the camera). In this full-body illusion version, subjects saw the synchronous stroking in front of the camera, inducing the experience of being at the position of the camera that was behind the subjects' body (illusory self-identification; Figure 3d). This shows that with slightly different manipulations of how visual and tactile stroking is applied and projected, MPS can be manipulated in a predictable fashion. Both studies revealed visual capture by showing that the location of the visual stroking dominates over the location of tactile stroking, leading in both cases to abnormal self-identification and, in the study of Lenggenhager et al., also to abnormal self-location $[38,57]$. Accordingly, abnormal self-identification coincided with the location of the visual information of the stroking and corresponds either to the visual body representation (virtual body [13]) or to the origin of the visual weak 1PP (camera [28]). Although more experimental work is needed, these data indicate that the experience of selflocation in terms of MPS can be separated experimentally from the experience of the spatial origin of the visual weak $1 \mathrm{PP}[13,57]$ and from the location of the visual body $[28,57]$. This is compatible with clinical data $[23,38,51,58]$ in which both types of dissociations have been observed in patients with heautoscopy or out-of-body experiences (i.e. a patient [51] experienced the visual weak $1 \mathrm{PP}$ at the position of his body, but self-location $\sim 2 \mathrm{~m}$ behind his body as represented). From the definition of out-of-body experiences (Box 2) it also follows that both study protocols did neither induce an out-of-body experience nor an autoscopic hallucination, but rather a state that is comparable to heautoscopy. Both study protocols enabled the manipulation of the key MPS-dimensions self-identification, self-location or weak 1PP, yet neither of them managed to manipulate what we have called the strong $1 \mathrm{PP}$ that is disturbed in heautoscopy and out-of-body experience. We predict that methodological approaches using multiple audio-visuo-spatial perspectives, multiple and spatially incongruent body positions, might lead to experimental effects beyond those described earlier and also involve the strong 1PP (maybe even aspects of the cognitive 1PP). This might be further facilitated through the more extensive use of virtual reality technology [59-62] and perhaps through repeated and prolonged exposure to such artificial bodily signals as championed by G.M. Stratton [54] over a century ago.

Although it is obviously not necessary to master concepts (such as the first-person pronoun 'I') or symbolic language to enjoy MPS, many recent authors have thought that agency is a necessary condition to actualize MPS. The experience of being the author of one's actions and to be able to selectively control a body at will with the help of explicit goal-repres- entations certainly is a central enabling condition for the development of MPS [11]. However, the reviewed clinical and experimental data show how a passive, multisensory and globalized experience of 'owning' a body is sufficient for minimal conscious selfhood. If, as we propose, 'global owning' - functionally defined as availability of an integrated, transparent and global representation of the spatiotemporally situated body - is the simplest form of self-consciousness, then it follows that MPS is constituted by something 'less' than agency. Global motor control or cognitive selfreference are not necessary conditions. We speculate that for the transition from MPS to the strong 1PP, attentional access [26] is necessary. Agency might be a sufficient condition for MPS, but it does not belong to the metaphysically necessary part of the minimal supervenience base for phenomenal selfhood, the set of properties which 'constitutes' our scientific target phenomenon. These and future experimental findings (Box 1) about MPS and related non-conceptual aspects of the self-model and their neurofunctional correlates might change the way we think about higher-levels of conscious self-representation, about the strong and cognitive 1PP, and the notion of 'a' self, as such. For now, our main point is that future research should concentrate on isolating the 'minimal' form of self-consciousness, and that full-body illusions are an interesting new entry point for research. A first step has already been taken: bodily agency is a causally enabling, but not a constitutive condition, for phenomenal selfhood.

\section{Acknowledgements}

T.M. is supported by a Fellowship at the Wissenschaftskolleg zu Berlin 2008/2009 and the Cogito Foundation. O.B. is supported by the Sandoz Family Foundation, the Cogito Foundation and the Swiss National Science Foundation.

\section{References}

1 Bermúdez, J.L. et al., eds (1995) The Body and the Self, MIT Press

2 Gallagher, S. (2005) How the Body Shapes the Mind. Oxford University Press

3 Baker, L. (1998) The first-person perspective: a test for naturalism. Am. Philos. Q. 35, 327-348

4 Bermúdez, J.L. (1998) The Paradox of Self-Consciousness. MIT Press

5 Gallagher, S. and Meltzoff, A.N. (1996) The earliest sense of self and others: Merleau-Ponty and recent developmental studies. Philos. Psychol. 9, 211-233

6 Metzinger, T. (2003) Being No One. The Self-Model Theory of Subjectivity. MIT Press

7 Metzinger, T. (2003) Phenomenal transparency and cognitive selfreference. Phenom. Cogn. Sci. 2, 353-393

8 Metzinger, T. (2008) Empirical perspectives from the self-model theory of subjectivity: a brief summary with examples. Prog. Brain Res. 168, $215-245$

9 Metzinger, T. and Gallese, V. (2003) The emergence of a shared action ontology: building blocks for a theory. Conscious. Cogn. 12, 549-571

10 Roessler, J.E.N. (2005) Agency and Self-Awareness. Oxford University Press

11 Pacherie, E. (2008) The phenomenology of action: a conceptual framework. Cognition 107, 179-217

12 Botvinick, M. and Cohen, J. (1998) Rubber hands 'feel' touch that eyes see. Nature 391, 756

13 Lenggenhager, B. et al. (2007) Video ergo sum: manipulating bodily self-consciousness. Science 317, 1096-1099

14 Nigro, G. and Neisser, U. (1983) Point of view in personal memories. Cognit. Psychol. 15, 467-482

15 Brugger, P. (2002) Reflective mirrors: perspective-taking in autoscopic phenomena. Cogn. Neuropsychiatry 7, 179-194

16 Vogeley, K. and Fink, G.R. (2003) Neural correlates of the first-personperspective. Trends Cogn. Sci. 7, 38-42 
17 Vogeley, K. et al. (2004) Neural correlates of first-person perspective as one constituent of human self-consciousness. J. Cogn. Neurosci. 16, 817-827

18 Arzy, S. et al. (2006) Neural basis of embodiment: distinct contributions of temporoparietal junction and extrastriate body area. J. Neurosci. 26, 8074-8081

19 Corradi-Dell'acqua, C. et al. (2008) Effects of shifting perspective of the self: an fMRI study. Neuroimage 40, 1902-1911

20 Damasio, A.R. (1999) The Feeling of What Happens: Body and Emotion in the Making of Consciousness. Harcourt Brace \& Company

21 Metzinger, T. (2004) Précis: being no one. In PSYCHE - An Interdisciplinary Journal of Research on Consciousness, 11, 1-35

22 Metzinger, T. (2006) Conscious volition and mental representation: towards a more fine-grained analysis. In Disorders of Volition (Sebanz, N. and Prinz, W., eds), pp. 19-48, MIT Press

23 Blanke, O. et al. (2004) Out-of-body experience and autoscopy of neurological origin. Brain 127, 243-258

24 Metzinger, T. (2005) Out-of-body experiences as the origin of the concept of a "soul". Mind and Matter 3, 57-84

25 Blanke, O. et al. (2002) Stimulating illusory own-body perceptions. Nature 419, 269-270

26 Blanke, O. and Castillo, V. (2007) Clinical neuroimaging in epileptic patients with autoscopic hallucinations and out-of-body experiences. Case report and review of the literature. Epileptologie 24, 90-96

27 Blanke, O. and Mohr, C. (2005) Out-of-body experience, heautoscopy, and autoscopic hallucination of neurological origin Implications for neurocognitive mechanisms of corporeal awareness and selfconsciousness. Brain Res. Brain Res. Rev. 50, 184-199

28 Ehrsson, H.H. (2007) The experimental induction of out-of-body experiences. Science 317, 1048

29 Gerstmann, J. (1942) Problem of imperception of disease and of impaired body territories with organic lesions. Relation to body schema and its disorders. Arch. Neurol. Psychiatry 48, 890-913

30 Halligan, P.W. et al. (1995) Unilateral somatoparaphrenia after right hemisphere stroke: a case description. Cortex 31, 173-182

31 Dieguez, S. et al. Méconnaissances corporelles perceptives et cognitives: la neurologie du schéma corpoel. Rev. Med. Suisse (in press)

32 Armel, K.C. and Ramachandran, V.S. (2003) Projecting sensations to external objects: evidence from skin conductance response. Proc. Biol. Sci. 270, 1499-1506

33 Tsakiris, M. and Haggard, P. (2005) The rubber hand illusion revisited: visuotactile integration and self-attribution. J. Exp. Psychol. 31, 80-91

34 Ehrsson, H.H. et al. (2004) That's my hand! Activity in premotor cortex reflects feeling of ownership of a limb. Science 305, 875-877

35 Tsakiris, M. et al. (2007) Neural signatures of body ownership: a sensory network for bodily self-consciousness. Cereb. Cortex 17, 2235-2244

36 Tastevin, J. (1937) En partant de l'expérience d'Aristote: les déplacements artificiels des parties du corps ne sont pas suivis par le sentiment de ces parties ni pas les sensations qu'on peut y produire. Encephale 1, 140-158

37 Brugger, P. (2006) From phantom limb to phantom body. Varieties of extracorporeal awareness. In Human Body Perception from the Inside Out (Knoblich, G. et al., eds), pp. 171-209, Oxford University Press

38 Blanke, O. et al. (2008) Multiple self locations and perspectives. Science E-letters (http://www.sciencemag.org/cgi/eletters/317/5841/1096)

39 Gardner, E.P. (1988) Somatosensory cortical mechanisms of feature detection in tactile and kinesthetic discrimination. Can. J. Physiol. Pharmacol. 66, 439-454
40 Maravita, A. and Iriki, A. (2004) Tools for the body (schema). Trends Cogn. Sci. 8, 79-86

41 Taoka, M. et al. (2000) Bilateral receptive field neurons in the hindlimb region of the postcentral somatosensory cortex in awake macaque monkeys. Exp. Brain Res. 134, 139-146

42 Graziano, M.S. et al. (2000) Coding the location of the arm by sight. Science 290, 1782-1786

43 Duhamel, J.R. et al. (1998) Ventral intraparietal area of the macaque: congruent visual and somatic response properties. J. Neurophysiol. 79, 126-136

44 Duhamel, J.R. et al. (1991) Congruent representations of monkey ventral intra-parietal cortex (area VIP). In Brain and Space (JPaillard, J. ed.), pp. 223-236, Oxford University Press

45 Lopez, C. et al. (2008) Body ownership and embodiment: vestibular and multisensory mechanisms. Neurophysiol. Clin. 38, 149-161

46 Lopez, C. and Blanke, O. (2007) Neuropsychology and Neurophysiology of Self-Consciousness. Multisensory and vestibular mechanisms. In Hirnforschung und Menschenbild. Beiträge zur interdisziplinären Verständigung (Holderegger, A., Sitter-Liver, B., Hess, C.W. and Rager, G., eds), pp. 183-206, Academic Press

47 Blanke, O. et al. (2008) Illusory reduplications of the human body and self. Handbook of Clinical Neurology 88, 429-458

48 Devinsky, O. et al. (1989) Autoscopic phenomena with seizures. Arch. Neurol. 46, 1080-1088

49 Brugger, P. et al. (1997) Illusory reduplication of one's own body: phenomenology and classification of autoscopic phenomena. Cogn. Neuropsychiatry 2, 19-38

50 Brugger, P. et al. (1994) Heautoscopy, epilepsy, and suicide. J. Neurol. Neurosurg. Psychiatry 57, 838-839

51 De Ridder, D. et al. (2007) Visualizing out-of-body experience in the brain. N. Engl. J. Med. 357, 1829-1833

52 Pearson, J. and Dewhurst, K. (1954) Sur deux cas de phénomenes héautoscopiques consecutifs à lésions organiques. Encephale 43, 166172

53 Lunn, V. (1970) Autoscopic phenomena. Acta Psychiatr. Scand. 46, $118-125$

54 Stratton, G.M. (1899) The spatial harmony of touch and sight. Mind 8, $492-550$

55 Mizumoto, M. and Ishikawa, M. (2005) Immunity to error through misidentification and the bodily illusion experiment. J. Conscious. Stud. 12, 3-19

56 Altschuler, E.L. and Ramachandran, V.S. (2007) A simple method to stand outside oneself. Perception 36, 632-634

57 Meyer, K. (2008) How does the brain localize the self? Science E-letters (http://www.sciencemag.org/cgi/eletters/317/5841/1096\#10767)

58 Schwabe, L. and Blanke, O. (2008) Phenomenology as another toolbox for neuroscientists? AbstrActa 2, 71-85

59 Tarr, M.J. and Warren, W.H. (2002) Virtual reality in behavioral neuroscience and beyond. Nat. Neurosci. 5, 1089-1092

60 Sanchez-Vives, M.V. and Slater, M. (2005) From presence to consciousness through virtual reality. Nat. Rev. Neurosci. 6, 332-339

61 Riva, G. (2007) Virtual reality and telepresence. Science 318, 12401242

62 Riecke, B.E. (2008) Consistent left-right reversals for visual path integration in virtual reality: more than a failure to update one's heading? Presence Teleoper. Virtual Environ. 17, 143-175

63 Shoemaker, S. (1968) Self-reference and self-awareness. J. Philos. 65, $555-567$ 